\title{
Universiteit
}

Leiden

The Netherlands

\section{Cell division and DNA segregation in Streptomyces: how to build a septum in the middle of nowhere? \\ Jakimowicz, D.; Wezel, G.P. van}

\section{Citation}

Jakimowicz, D., \& Wezel, G. P. van. (2012). Cell division and DNA segregation in Streptomyces: how to build a septum in the middle of nowhere? Molecular Microbiology, 85(3), 393-404. doi:10.1111/j.1365-2958.2012.08107.x

Version: $\quad$ Publisher's Version

License: $\quad$ Leiden University Non-exclusive license

Downloaded from: https://hdl.handle.net/1887/3238769

Note: To cite this publication please use the final published version (if applicable). 


\section{Cell division and DNA segregation in Streptomyces: how to build a septum in the middle of nowhere?}

Dagmara Jakimowicz ${ }^{1,3}$ and Gilles P. van Wezel ${ }^{2 *}$ ${ }^{1}$ Faculty of Biotechnology, University of Wroclaw, UI. Tamka 2, 50-137 Wroclaw, Poland.

${ }^{2}$ Molecular Biotechnology, Sylvius Laboratories, Leiden University, PO Box 9505, 2300RA Leiden, the

Netherlands.

${ }^{3}$ Institute of Immunology and Experimental Therapy, Weigla 12, 53-114 Wroclaw, Poland.

\section{Summary}

Streptomycetes are antibiotic-producing filamentous microorganisms that have a mycelial life style. In many ways streptomycetes are the odd ones out in terms of cell division. While the basic components of the cell division machinery are similar to those found in rodshaped bacteria such as Escherichia coli and Bacillus subtilis, many aspects of the control of cell division and its co-ordination with chromosome segregation are remarkably different. The rather astonishing fact that cell division is not essential for growth makes these bacteria unique. The fundamental difference between the cross-walls produced during normal growth and sporulation septa formed in aerial hyphae, and the role of the divisome in their formation are discussed. We then take a closer look at the way septum site localization is regulated in the long and multinucleoid Streptomyces hyphae, with particular focus on actinomycete-specific proteins and the role of nucleoid segregation and condensation.

\section{Introduction}

Streptomycetes are multicellular Gram-positive bacteria that are of great importance for biotechnology for their ability to produce a large array of natural products, including antibiotics, anticancer agents and immunosuppressants, as well as industrial enzymes (Hopwood, 2007). The life cycle starts with a single spore that germinates to form

Accepted 18 May, 2012. *For correspondence. E-mail g.wezel@ chem.leidenuniv.nl; Tel. (+31) 71 5274310; Fax (+31) 715274340. young vegetative hyphae, which then grow out following a process of hyphal growth and branching, to produce a branched vegetative (substrate) mycelium resembling that of filamentous fungi (Chater and Losick, 1997). A prominent feature of the vegetative hyphae of Streptomyces is their growth by tip extension, i.e. new cell wall material is incorporated at the tips of apical cells, and the control of division should be viewed in this context (Flärdh, 2003a). Exponential growth of the vegetative mycelium is achieved by combination of tip growth and branching, rather than by division. In contrast, during vegetative growth cell division results in the formation of hyphal cross-walls, which are widely spaced, and delimit adjacent elongated compartments that each contain multiple copies of the genome. Studies of Streptomyces granaticolor suggested initiation of division at the middle of the growing apical cell when it reaches a certain length (Kretschmer, 1982). However, considerable variation is seen in the frequency and spacing of vegetative septa in Streptomyces hyphae, both between different species and within a single species between different growth conditions. This is in contrast to Escherichia coli, in which cell division occurs at the midcell, after daughter nucleoids have been segregated. Moreover, in non-actinomycete rod-shaped bacteria, cell elongation is due to synthesis of new cell wall material in the lateral walls, rather than at the tips (Donachie, 1993). At the time of nutrient depletion, the vegetative mycelium hyphae differentiate to form erected sporogenic structures called aerial hyphae. The production of antibiotics is temporally correlated to this phase of the Streptomyces life cycle (Bibb, 2005; van Wezel and McDowall, 2011). The aerial hyphae give the colonies their characteristic white and fluffy appearance and are eventually converted into chains of unigenomic spores (Chater and Losick, 1997). Genes that are required for the formation of aerial hyphae are referred to as bld, referring to the bald ('hairless') phenotype of the mutants lacking the fluffy aerial hyphae (Merrick, 1976).

Aerial hyphae differ from vegetative hyphae for their lack of branches, width (approximately twice that of vegetative hyphae), fast elongation rate and intensive chromosome replication. In a full-length aerial hypha that has stopped 


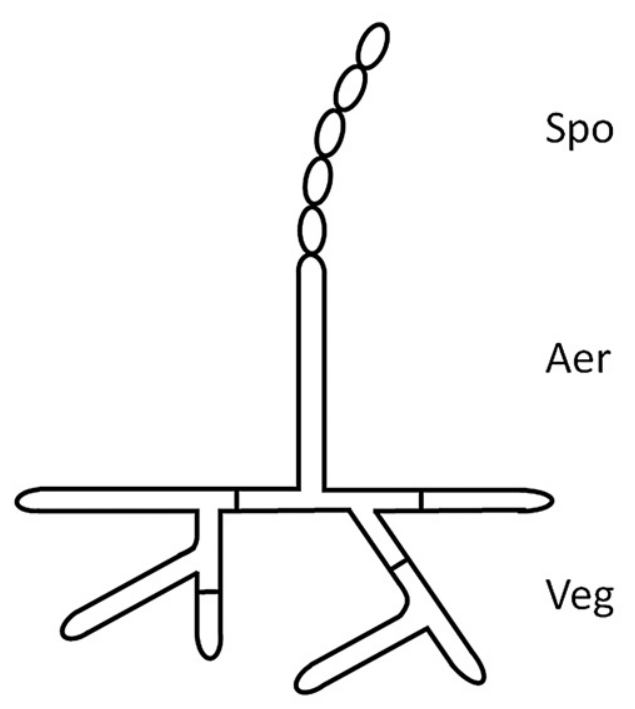

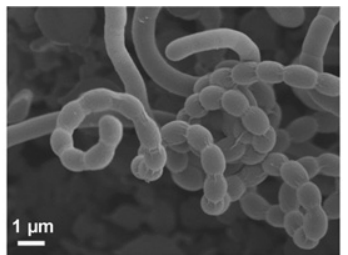

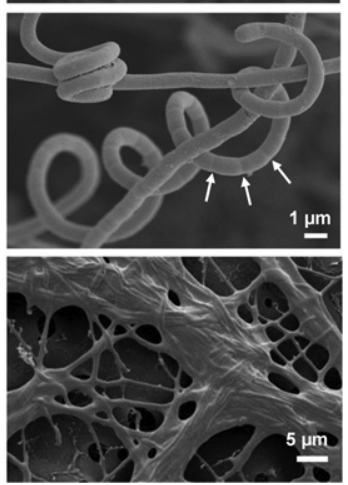

Fig. 1. Two different types of cell division in streptomycetes. Left, schematic drawing of the Streptomyces mycelium; right, cryo-scanning electron micrographs of vegetative mycelium (bottom), prespores (middle) and a mature spore chain (top). The vegetative hyphae (Veg) grow by tip extension and branching, and widely spaced cross-walls are produced at irregular intervals, which lead to a multicellular network. For a detailed image of a cross-wall see Fig. 2A. Aerial hyphae (Aer) are unbranched, and chains of spores (Spo) are formed during a

co-ordinated multiple cell division event in a dedicated sporogenic part of the aerial hyphae, with up to 100 septa formed simultaneously (arrows indicate indentations indicative of ongoing division). The sporogenic section may be separated from the non-sporogenic part by one or more basal septa (see Fig. 2B). growing, synchronous emergence of a ladder of uniformly spaced septa is observed, accompanied by condensation and segregation of numerous chromosomes, and the conversion of the aerial hyphae into prespores. Although the process of chromosome segregation and septation in Streptomyces aerial hyphae involves many homologues of proteins also found in other bacteria, sporulation events require several Streptomyces-specific mechanisms, particularly in terms of regulation. An important set of sporulation regulators are those encoded by the whi genes, which are so-called for the lack of pigmentation of the mutant colonies (Chater, 1972); whi mutants cannot enter sporulation and thus fail to produce the grey spore pigment. The early whi genes control the expression of a number of sporulation-associated genes such as ftsZ, parAB, sigF, ssgAB and smeF (discussed below).

Many streptomycetes, including for example the streptomycin producer Streptomyces griseus and the chloramphenicol-producing Streptomyces venezuelae, also sporulate in submerged culture, whereby sporogenic hyphae produce short spore chains (Kendrick and Ensign, 1983; Glazebrook et al., 1990). Submerged sporulation has not been studied extensively, but is important for our understanding of the process of sporulation-specific cell division; it allows the identification of novel developmental regulators that specifically affect the ability to produce spores but do not influence aerial hyphae formation. One such gene is $s s g A$, originally identified in S. griseus (see below), as its overexpression induces fragmentation and even aberrant submerged sporulation in Streptomyces coelicolor (Kawamoto et al., 1997; van Wezel et al., 2000). The function of this gene in the control of cell division is discussed below.

\section{Two distinct modes of cell division in streptomycetes}

\section{Cross-walls and sporulation septa}

Sporulation septa are distinctly different in morphology and function from vegetative septa or cross-walls, and the occurrence of two distinct types of cell division makes Streptomyces rather unique (Fig. 1). The most determining feature is the fact that vegetative septa lead to the formation of compartments, but not to physical separation, with as a result the formation of a syncytial network of connected compartments - in other words, a truly multicellular bacterium (Wildermuth and Hopwood, 1970; Chater and Losick, 1997). Imaging of such cross-walls at high resolution sometimes reveals likely channels which may serve to allow transport of components over the membrane (Fig. 2A). Cryo-electron tomography, which was previously successfully applied to image the septal pore cap in free-substituted samples of basidomycetes (Muller et al., 1998; 2000) and to analyse the final stages of division in Caulobacter crescentus (Judd et al., 2005), should hopefully allow a three-dimensional reconstruction of a Streptomyces septum (K. Celler et al., unpublished).

In contrast, the sporulation septum is much thicker than the cross-wall and does lead to physical separation of cells, in casu chains of spores in aerial hyphae. Perhaps, there is also a third type of septum, sometimes referred to as the 'basal septum'. Work on submerged sporulation by $S$. griseus revealed the presence of a septum or crosswall at the base of sporogenic hyphae in submerged cultures, which may serve to delimit a part of the hyphae allowing increased expression of fts $Z$ and thus enable sporulation septation (Kwak et al., 2001). Such a septum was also found at the base of nascent aerial hyphae, and was proposed to serve to allow compartment-specific 
A
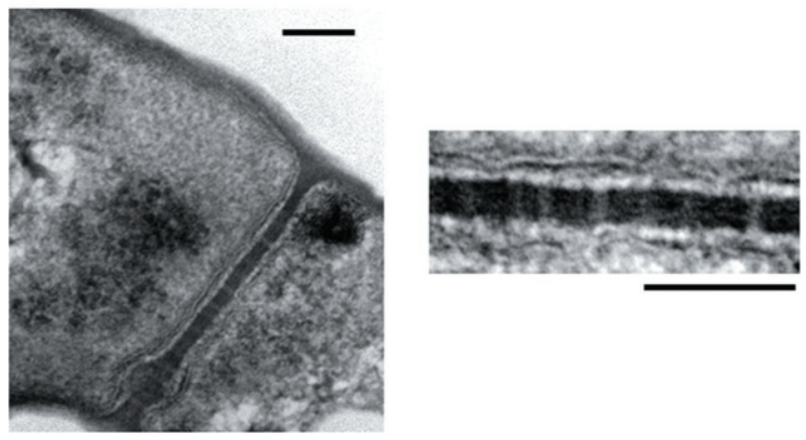

B
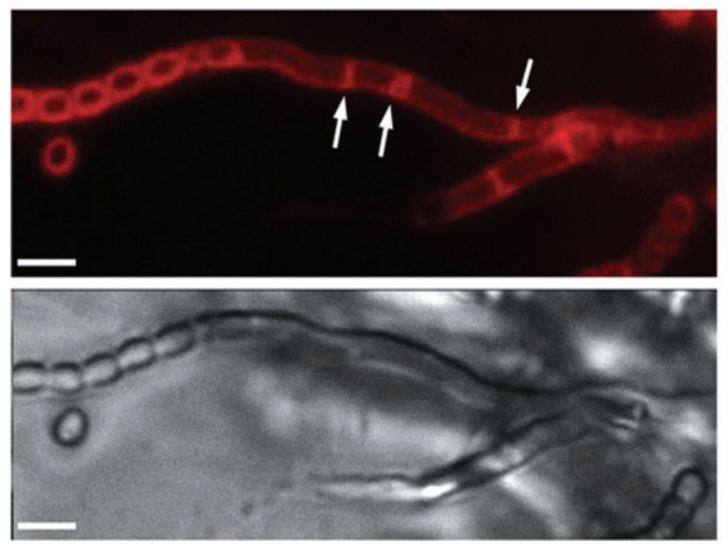

Fig. 2. Septation in vegetative and aerial hyphae of $S$. coelicolor. A. High-resolution transmission electron micrographs of a vegetative septum (so-called cross-wall) of $S$. coelicolor. Left, complete cross-wall with putative channels (apparent as lighter sections) and a bulge; right, close-up of part of the cross-wall. Bar, $100 \mathrm{~nm}$. It should be noted that cross-walls are not always uniform in width and appearance.

B. Fluorescence (top) and light (bottom) image of sporogenic aerial hyphae. Cross-walls are clearly visible in the non-sporulating part of the aerial hypha (arrows). Bar, $2 \mu \mathrm{m}$.

gene expression (Dalton et al., 2007). 'Basal' septa are readily detected close to mature spore chains, and also observed at the base of the aerial hypha (Fig. 2B). Interestingly, analysis of sporulation mutants (whi, ssgB) that are blocked in sporulation revealed multiple septa in aerial hyphae with similar spacing and width as vegetative cross-walls which - like in vegetative hyphae - do not lead to physical separation of the adjacent compartments (Grantcharova et al., 2005; Willemse et al., 2011). This seems to argue against the existence of a structurally distinct 'basal' aerial septum; rather, it may well be that cross-walls are part and parcel of aerial growth.

\section{Requirement of the canonical divisome for growth and septation}

The mycelial life style of streptomycetes makes them rather exceptional microorganisms in terms of cell division. This is not so much in make-up of the cell division machinery itself, but rather in the way that septum site localization is controlled and how septum formation and chromosome segregation are co-ordinated (Table 1). Perhaps, the most striking feature of streptomycetes is that cell division is not essential for growth of the hyphae (McCormick et al., 1994; McCormick, 2009), which makes Streptomyces ideal organisms for the analysis of cell division proteins (see below). However, fts $Z$ null mutants fail to produce spores, so that they are unlikely to survive in the soil, their natural habitat.

Many of the cell division genes required for binary fission have been identified by analysing temperaturesensitive mutants that are defective in division [the term $\mathrm{fts}$, for filamentous temperature sensitive, was coined by Van de Putte et al. (1964)]. At non-permissive temperatures the fts mutants fail to divide and stop growing. Obviously, non-dividing rod-shaped cells only display linear growth, and a yet unknown mechanism prevents filamentous E. coli or Bacillus subtilis cells from producing very long filaments. One explanation may be that in contrast to Streptomyces hyphae, the cell wall of rod-shaped bacteria is not designed to sustain filamentous growth. Filamentous mutants of $E$. coli that are able to divide occasionally are viable, forming small colonies with merely long filamentous cells (Van de Putte et al., 1964).

Table 1. Conceptual differences in cell division and DNA segregation between rod-shaped bacteria and streptomycetes.

Rod-shaped bacteria

Cell division is essential for growth

Cell division results in physical separation of cells

A single septum is produced at mid-cell or during sporulation (in the case of Bacillus)

FtsZ localization is negatively controlled by among others the Min system excluding FtsZ at non-division sites

Growth by lateral cell wall extension in most bacteria

Nucleoid occlusion prevents septum formation over non-segregated chromosomes

\section{Streptomycetes}

Cell division is essential for sporulation but not for viability

Cell division physically separates cells during sporulation; cross-walls in vegetative hyphae produce connected compartments

Multiple septa produced simultaneously during sporulation; irregularly spaced cross-walls produced during vegetative growth

During sporulation FtsZ is recruited by SsgB; mechanism during vegetative growth unknown

Hyphae grow by tip extension

Nucleoid occlusion-like mechanism unknown; the nucleoid may pay a role in determining the septum position

For references, see text. 
In the mycelial network of streptomycetes, exponential growth is achieved by tip growth and branching, neither of which are blocked in the absence of cell division. Interestingly, the mycelia of fts $Z$ null mutants can be fragmented, suggesting that somehow the contents are prevented from spilling out. Preliminary evidence points at the possibility that the space between septa is not contiguous as believed so far, but may in fact be divided in smaller compartments (J. Willemse and G.P. van Wezel, unpublished). This would explain the viability of fragmented fts $Z$ mutants.

The inventory of the cell division machinery in streptomycetes highlights many similarities to the basic mechanism of cell division found in most other eubacteria. The cell division protein FtsZ polymerises under GTP hydrolysis into protofilaments, which assemble to constitute a dynamic ring-like structure known as the Z-ring ( $\mathrm{Bi}$ and Lutkenhaus, 1991; de Boer et al., 1992; Erickson, 1995). This Z-ring then mediates the recruitment of the cell division machinery (divisome) components to the mid-cell position (reviewed in Goehring and Beckwith, 2005; Adams and Errington, 2009) and provides the driving force for constriction during cell division (Errington et al., 2003; Erickson et al., 2010). It was suggested that FtsW may play a role in stabilization of the Z-ring in streptomycetes, although how this should be brought about is yet unknown (Mistry et al., 2008). Besides fts $Z$ itself, other cell division genes are also dispensable for growth, and null mutants have been made for among others divIC, fts $L$ (Bennett et al., 2007), ftsl, ftsW (Mistry et al., 2008; Bennett et al., 2009), ftsE and ftsX (Noens, 2007; McCormick, 2009), and ftsQ (McCormick and Losick, 1996). For details on the divisome of streptomycetes we refer to the literature (Flärdh and van Wezel, 2003; McCormick, 2009).

Importantly, while fts $Z$ null mutants are completely devoid of septa, deletion of other cell division genes primarily affects sporulation-specific cell division. This is true for several fts mutants, e.g. deleted for ftsW and ftsl (Mistry et al., 2008), as well as for mutants lacking ssgB required for recruitment of FtsZ during sporulation-specific cell division (Willemse et al., 2011; see below). Indeed, knocking out fts genes has a highly variable effect in S. coelicolor, ranging from almost complete lack of septa in $f t s Q$ mutants to medium-dependent phenotypes for many other cell division mutants (see references above; reviewed in McCormick, 2009). The rather baffling implication of this is that while FtsZ ring formation is required for cross-wall formation, the other divisome components are not. The explanation for this may be found in the fact that the divisome is directed at performing cell-separating cytokinesis, with activation of Z-ring contraction, while this is undesirable when cross-walls are formed. Furthermore, (some of) the cross-walls may contain channels (Fig. 2A), and it is difficult to see how these would be implemented via canonical divisome-directed division. Modelling and biophysical studies support the idea that high-level expression of FtsZ may be sufficient for self-assembly and Z-ring formation (Navajas et al., 2008; Kuchibhatla et al., 2009). These interesting concepts deserve further investigation.

\section{Control of septum site localization}

The mycelial life style of streptomycetes imposes novel requirements for cell division and its control mechanisms, e.g. due to the lack of a defined mid-cell position and apical growth. During sporulation-specific cell division, FtsZ initially forms long filaments in the aerial hyphae, followed by localization in a focal pattern during the onset of cell division and finally by Z-ring formation, visible as spectacular FtsZ ladders that are typical of streptomycetes (Schwedock et al., 1997; Grantcharova et al., 2005; Willemse and van Wezel, 2009). Not surprisingly, the way cell division is controlled is also remarkably different. In rod-shaped bacteria several proteins are required to assist in septum site localization and to stabilize the Z-ring, including FtsA and ZipA (Hale and de Boer, 1997; RayChaudhuri, 1999; Pichoff and Lutkenhaus, 2002), ZapA (Gueiros-Filho and Losick, 2002) and SepF (Hamoen et al., 2006). The positioning and timing of septum formation typically involves the action of negative control systems such as Min, which prevents Z-ring assembly at the cell poles (Raskin and de Boer, 1997; Marston et al., 1998), and nucleoid occlusion that prevents formation of the Z-ring over non-segregated chromosomes (Woldringh et al., 1991; Wu and Errington, 2004; 2011; Bernhardt and de Boer, 2005). Direct homologues of these control proteins are missing in streptomycetes. Perhaps, most illustrative of the major difference between division in hyphae and in unicellular bacteria is that SepF and DivIVA are the only Streptomyces homologues of septum localization and control systems found in other bacteria, and at least for DivIVA (an orthologue of MinE) it is clear that it plays a different role; in this organism, DivIVA is not involved in the control of division, but is instead required for driving apical (tip) extension and is therefore essential for growth (Flärdh, 2003b). Literature reiterates the putative presence of two $\min D$ homologues (and some databases even refer to more $\min D$ genes), but these have only remote sequence similarity to $\min D$ and are in fact part of operons encoding type II/type V secretion systems involved in the formation of fimbriae-like structures (G.P. van Wezel, unpublished). Deletion of these genes, either as single mutants or in combination, does not notably affect cell division (G.P. van Wezel and J. McCormick, unpublished). S. coelicolor has two sepFparalogues which have hitherto not been studied. FtsZ and the cytokinetic Z-ring, as well as a number of 
membrane proteins likely involved in linking the Z-ring to synthesis of septal peptidoglycan, are present. Detailed characterization of the divisome has been performed elsewhere (Flärdh and van Wezel, 2003; McCormick, 2009).

\section{Actinomycete-specific proteins that govern cell division and hyphal stability}

With the remarkable existence of two distinct cell division structures in a single organism, the logical questions that arise are, how are cross-wall formation and sporulationspecific cell division orchestrated, and which proteins control the localization of the septa at these different stages of growth? This seems rather haphazard for vegetative cross-walls, while the many septa formed during sporulation-specific cell division are all positioned at exactly the same distance, so as to produce the very distinctive FtsZ ladders (Schwedock et al., 1997). Considering the absence of the canonical cell division control proteins, we need to search for actinomycete-specific proteins. Before we do that, it is important to note that FtsZ itself plays a major role in enabling sporulationspecific cell division. Either reduced expression of $\mathrm{fts} Z$ following deletion of the developmental ftsZp2 promoter (Flärdh et al., 2000) or a missense mutation in the FtsZ protein itself (Grantcharova et al., 2003) abolishes sporulation-specific cell division, while vegetative cell division is not affected. Indeed, the notion that the expression of FtsZ is a major determinant for the switch to aerial division is supported by the observation that sporulation could be restored to each of the six early whi mutants (whiA, $B, G, H, I, \mathcal{J}$ ) by placing fts $Z$ behind a constitutive promoter (Willemse et al., 2012). This indicates that a major function of the Whi proteins in terms of the intrinsic capacity to sporulate is to ensure the proper timing and level of FtsZ production.

CrgA is a member of a novel family of small proteins in actinomycetes, and affects sporulation-specific cell division, by influencing Z-ring assembly via the control of FtsZ turnover (Del Sol et al., 2006). S. coelicolor crgA null mutants show premature development of aerial hyphae on glucose-containing media and produce swollen spores not dissimilar to those formed by mreB mutants (Mazza et al., 2006), as well as a significant increase in the abundance of FtsZ rings (Del Sol et al., 2006). CrgA also occurs in non-sporulating actinomycetes, and in Mycobacterium it was identified as a divisome component that interacts with the cell division proteins FtsZ, Ftsl and FtsQ (Plocinski et al., 2011). Two-hybrid studies revealed that CrgA also interacts with FtsQ and Ftsl in S. coelicolor (P. Dyson, unpublished). Deletion of $\operatorname{crg} A$ in Mycobacterium smegmatis reduced growth rate and led to the formation of bulged cells, again suggesting a relationship to cytoskeleton and cell shape maintenance (Plocinski et al.,
2011). The phenotypes of $\operatorname{crg} A$ null mutants and of strains overexpressing the protein suggests it affects both cell division and the cytoskeleton. Streptomycetes likely have an extensive cytoskeleton, based on the number of large proteins with coiled-coil structural elements and the emerging evidence of their involvement in cell shape and morphogenesis (Mazza et al., 2006; Bagchi et al., 2008; Walshaw et al., 2010; Heichlinger et al., 2011; K. Celler, J. Willemse and G.P. van Wezel, unpublished). For instance, intermediate filament-like structures are formed by the FilP protein (SCO5396), which contribute significantly to the mechanical resilience of the hyphae of $S$. coelicolor (Bagchi et al., 2008). The precise function of the many coiled-coil proteins in the growth and stability of the Streptomyces hyphae remains to be elucidated.

\section{The SsgA-like proteins}

Another actinomycete-specific family of proteins that plays an important role in morphogenesis and control of cell division is that of the SsgA-like proteins (SALPS). Members of this family are typically between 130 and 145 aa long, with $30-50 \%$ aa identity between them. SALPs occur exclusively in morphologically complex actinomycetes, and there is a suggestive linkage between the number of paralogues and the complexity of the developmental process in actinobacteria (Traag and van Wezel, 2008). S. coelicolor contains seven SALPs (SsgA-G; Noens et al., 2005), of which the cell division-related SsgA, SsgB and SsgG are most relevant here.

SsgA (for sporulation of Streptomyces griseus A) was identified as a possible sporulation protein in $S$. griseus (Kawamoto and Ensign, 1995), activating septum formation in vegetative hyphae, which results in fragmentation of mycelia in liquid-grown cultures (Kawamoto et al., 1997; van Wezel et al., 2006). SsgA is required for both solid- and liquid-culture sporulation of streptomycetes and is controlled by the autoregulatory molecule A factor in S. griseus (Jiang and Kendrick, 2000; van Wezel et al., 2000; Yamazaki et al., 2003). It is therefore a key connection between the two types of cell division. Transcription of $s s g A$ does not depend on the whi sporulation genes, but instead depends on the IcIR family regulator SsgR encoded by the upstream located $s s g R$ gene, most likely to allow separation of its function during vegetative and aerial growth (Traag et al., 2004).

SsgA localizes to the sites where cell wall remodelling takes place; while it was shown to activate germination and cell division, its precise role in tip growth and branching is unclear (Noens et al., 2007). Microarray studies revealed that close to 1000 genes were more than twofold deregulated in an ssgA null mutant of $S$. coelicolor, including almost all developmental genes (bld, whi), as well as divIVA, ftsl and many genes involved in DNA segregation 


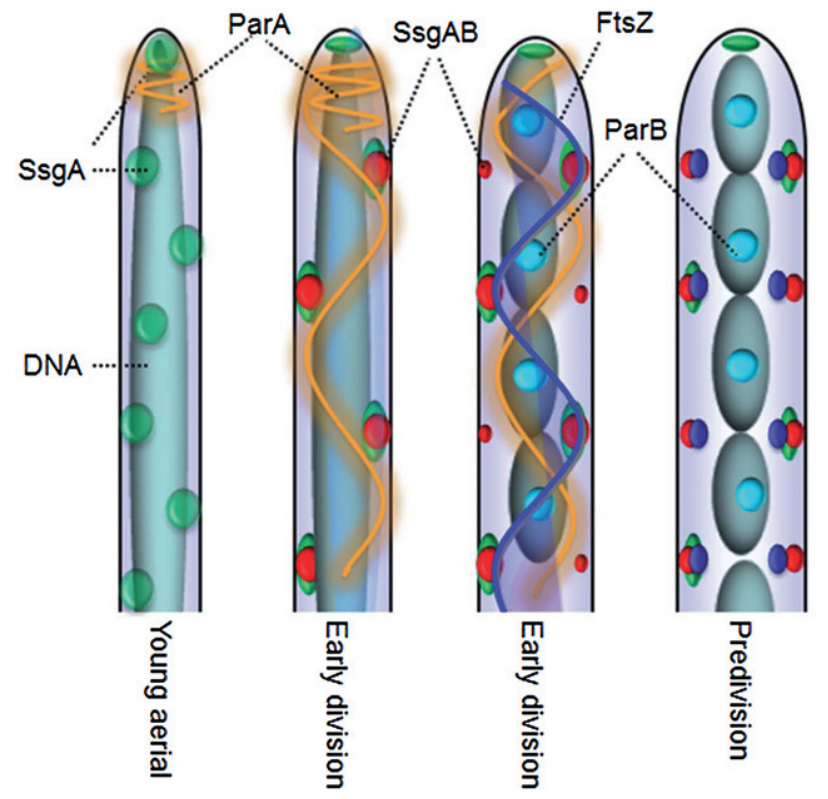

and topology (Noens et al., 2007). The remarkable upregulation of these genes is probably explained as an attempt to compensate for the absence of SsgA.

\section{$S s g B$ and the recruitment of Fts $Z$}

SsgB is most likely the archetype of the SALPs and the crystal structure of SsgB from Thermobifida fusca was determined at $2.6 \AA$ resolution (Xu et al., 2009). This revealed a bell-shaped trimer with intriguing structural similarity to the mitochondrial guide RNA-binding proteins MRP1 and MRP2 (Schumacher et al., 2006) and the ssDNA-binding protein PBF-2 (Desveaux et al., 2002). The $\mathrm{SsgB}$ protein is required for sporulation (Keijser et al., 2003; Sevcikova and Kormanec, 2003) and part of the divisome, where it colocalizes with FtsZ during the entire process of sporulation-specific cell division (Willemse et al., 2011). Interestingly, during early aerial growth - prior to the onset of division - SsgB foci are formed while FtsZ localization is still diffuse, and live imaging showed that FtsZ localizes specifically to SsgB foci. The SsgB localization pattern in young aerial hyphae is very similar in wildtype aerial hyphae and those of fts $Z$ null mutants, with foci at around $1 \mu \mathrm{m}$ distance along the hyphal wall. Förster resonance energy transfer combined with fluorescence lifetime imaging (FRET-FLIM) revealed that SsgB indeed interacts closely with FtsZ and with the membrane (Willemse et al., 2011). FRET-FLIM is almost exclusively applied in eukaryotic cell biology and a very powerful tool for the in vivo imaging and calculation of distances between proteins or between a protein and other cellular components such as the membrane, cell wall or DNA

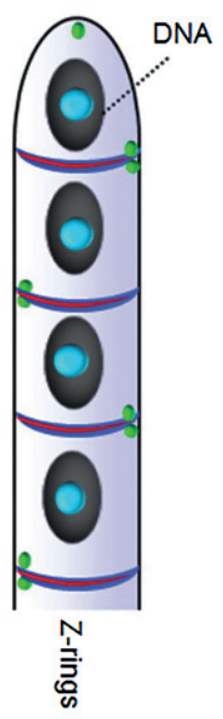

Fig. 3. Model for the control of Z-ring formation in Streptomyces. In young aerial hyphae, SsgA localizes in a highly dynamic focal pattern while SsgB and FtsZ are still diffuse. At that time ParA is mainly constrained to the hyphal tip. Then in early division stages SsgA and SsgB colocalize temporarily at typically alternating sides of the aerial hyphae, while ParA extends as filaments along the hyphae. In the presence of ParA, ParB complexes are formed over the yet uncondensed DNA. Prior to sporulation-specific cell division, FtsZ assembles in spiral-like filaments through the hyphae. From this point onwards in predivision hyphae, FtsZ and SsgB continue to colocalize, whereby SsgB tethers FtsZ to the membrane. Z-rings are then formed at the sporulation stage, followed by chromosome condensation and segregation and formation of the sporulation septa. When spores mature SsgA localizes at either side of the septa and marks the future germination sites. Figure adapted from Willemse et al. (2011).
(Miyawaki et al., 1997; Chen et al., 2003); the addition of a FLIM component makes application of FRET in bacterial cells feasible (van Munster and Gadella, 2005; Alexeeva et al., 2010; Willemse et al., 2011). Two-hybrid studies further showed that in fact SsgB with the N-terminal domain of FtsZ, and the requirement of this interaction may explain why substituting the fts $Z$ gene from $S$. coelicolor by that of Mycobacterium does not support cell division in $S$. coelicolor, while fts $Z^{M t u b}$ can substitute the fts $Z$ orthologue of E. coli, which has much lower overall homology (McCormick, 2009).

SsgB activates the polymerization of FtsZ protofilaments in vitro, resulting in long FtsZ filaments of around $450 \mathrm{~nm}$ (Willemse et al., 2011). Different modes of action have been described for proteins involved in FtsZ filament formation, such as ZipA that stimulates the formation of filament networks (RayChaudhuri, 1999), or ZapA that promotes bundling of the filaments (Gueiros-Filho and Losick, 2002; Low et al., 2004). The mode of action of $\mathrm{SsgB}$ is similar to that of ZipA.

These observations have several important general implications. First, FtsZ localization is not the result of negative control systems such as MinCDE, but rather is positively controlled by direct recruitment. Second, in contrast to the canonical systems such as those studied in E. coli and B. subtilis, FtsZ is not the first divisome component to arrive at the septum site. This implies the evolution of an entirely different way of Z-ring control in actinomycetes, which may be explained by the absence of a mid-cell reference point in the long multinucleoid hyphae. A model for septum site localization and DNA segregation in Streptomyces is presented in Fig. 3. 


\section{Chromosome segregation and condensation}

In rod-shaped bacteria, as soon as the oriC regions (origins of chromosome replication) have been replicated they are actively transported towards the cell poles, while the remainder of the chromosomes continues to be replicated (recently reviewed in Toro and Shapiro, 2010). Chromosome segregation in Streptomyces, as in the other bacteria, involves ParA and ParB family proteins. ParA homologues are Walker A ATPases, which can oligomerize into filaments and show dynamic localization within the cell. ParA has been postulated to supply energy for the movement of ParB segregation complexes (Leonard et al., 2005a,b). ParB homologues are DNAbinding proteins that form higher-order nucleoprotein complexes at partitioning sites (called parS) near the origin of replication (Leonard et al., 2004; Hayes and Barilla, 2006). In Streptomyces hyphal compartments, multiple chromosomes remain uncondensed until the final stages of sporulation, and their replication in both vegetative and aerial hyphae seems asynchronous (Ruban-Osmialowska et al., 2006). In vegetative hyphae there does not seem to be a requirement for precise chromosome segregation, although the expression of the par genes at this stage of growth suggests their role at this growth phase. During elongation of the hyphae, replicating chromosomes follow the growing tip (Wolanski et al., 2011), and recent experiments suggest that perhaps $\mathrm{Par} A \mathrm{~B}$ are involved in chromosome anchorage at the tips (P. Herron, A. Kois-Ostrowska and D. Jakimowicz, unpublished). Moreover, ParA and ParB protein may be involved in the control of chromosome replication, since $B$. subtilis ParA directly affects the function of replication initiator DnaA (Murray and Errington, 2008; Scholefield et al., 2012). Such interaction has not been detected in Streptomyces, but ParB complexes formed close to the replication origin may provide steric hindrance for DnaA and in such a way regulate chromosome replication.

Synchronized condensation and segregation of dozens of chromosomes coupled to multiple septation in the sporulating aerial hyphae impose special requirements on the spatiotemporal regulation of proteins involved in chromosome organization. Chromosomes are condensed and segregated when their intensive replication slows down, coinciding with growth cessation of the aerial hyphae. While in rod-shaped bacteria ParB complexes, fuelled by ParA, actively transfer oriC regions towards the cell poles after replication, in Streptomyces they align chromosomes in regular intervals along the hyphae during condensation, so as to ensure that each prespore receives a copy of the chromosome. Assembly of ParB complexes is dependent on distinctive ParA structures, forming elongated filaments extending from the tip of the hyphae over tens of microns.
Deletion of parAB results in disturbed septation and chromosome segregation (Kim et al., 2000; Jakimowicz et al., 2005; 2007). Although Streptomyces ParA shares many features with its orthologues in rod-shaped bacteria, such as polymerization and stimulation of ATPase activity by ParB, it also exhibits unique properties. For example, ParA polymerization was shown to be affected by the actinobacteria-specific protein ParJ, which also affects chromosome segregation and septation during sporulation (Ditkowski et al., 2010). The interaction of ParA with ParJ apparently mediates the switch between hyphal growth and the onset of sporulation, and may be interlinked with polar growth, which is characteristic not only of streptomycetes, but also of non-sporulating actinobacteria. Co-ordination of ParA filamentation with aerial growth is dependent on interaction with cytoskeleton-like scaffolds at the growing tip (D. Jakimowicz and B. Ditkowski, unpublished).

Another important protein that affects the assembly of ParB complexes is SMC (for structural maintenance of chromosomes). As in other bacteria, SMC is required for efficient chromosome condensation in Streptomyces and a prerequisite for prespore formation (Dedrick et al., 2009; Kois et al., 2009) and the timing of its activity overlaps that of ParB-mediated segregation. However, deletion of $s m c$ aggravates the chromosome segregation defects in parB mutants, suggesting that SMC can function independent of ParB. The final stage of Streptomyces chromosome segregation is assisted by the cell division protein FtsK (Dedrick et al., 2009). In B. subtilis, the FtsK homologue SpolllE transports chromosomes thorough the closing septum during sporulation and forms a channel that partitions the daughter cell membranes, acting as a translocation complex at mid-cell (Burton et al., 2007; Fleming et al., 2010). SpollIE is required for sporulation in B. subtilis, while in Streptomyces the absence of its orthologue FtsK does not inhibit sporulation but instead leads to increased genetic instability, perhaps due to the incomplete transfer of chromosomes and hence DNA damage during septum closure (Wang et al., 2007). A second SpollIE/FtsK family protein, SffA, is also required for correct DNA segregation/ condensation in streptomycetes, and acts in concert with the small membrane protein SmeA (Ausmees et al., 2007). The proteins are expressed specifically in sporogenic aerial hyphae during divisome assembly. SffA accumulates specifically at sporulation septa where it colocalizes with FtsK, and its septal localization depends on SmeA. The exact way these interesting proteins control DNA segregation process is yet unknown.

Interestingly, there are variants of chromosome architectural proteins, in particular so-called nucleoid-associated proteins (NAP), which only occur in actinomycetes. An example of such proteins is HupS - one of the two Strep- 
tomyces HU paralogues, which is involved in compacting the DNA of the bacterial nucleoid (Pettijohn, 1988). Recent evidence suggests that $E$. coli $\mathrm{HU}$ may direct the spatial distribution of RNA polymerase in the nucleoid (Berger et al., 2010). In S. coelicolor HupA is the conventional HU protein. HupS is an actinomycete-specific two-domain protein, which, in addition to the $\mathrm{N}$-terminal $\mathrm{HU}$ domain, contains a C-terminal domain with homology to histone linker proteins in eukaryotes ( $\mathrm{H} 1$-like domain). Transcription of hupS is co-ordinated with sporulation, and the HupS protein plays a role in nucleoid compaction and enhanced heat resistance in spores (Salerno et al., 2009). The other NAP protein is integration host factor (IHF), which has essentially the same structural fold as $\mathrm{HU}$ (Rice et al., 1996) and plays an important role in site-specific recombination, DNA replication, transcription and genome organization in bacteria (Goosen and van de Putte, 1995). Three IHF homologues occur in streptomycetes. One of these, sIHF, belongs to an actinomycete-specific type of IHF that was first identified in mycobacteria, and is essential for their survival (Pedulla et al., 1996; Pedulla and Hatfull, 1998). S. coelicolor sihf mutants have strongly reduced viability, which may be explained by defective DNA segregation during sporulation (Yang et al., 2012). Additionally, chromosome condensation is positively controlled by Dps-type NAP, which belong to the ferritin proteins that control nucleoid structure during stationary phase in E. coli, and form large crystalline-like nucleoprotein complexes with the DNA (Nair and Finkel, 2004). In Streptomyces two of the three homologues (DpsA and DpsC) are developmentally controlled; they affect spore nucleoids and positioning of the sporulation septa (Facey et al., 2009), and present an example of actinomycete-specific architectural proteins that likely play a specific role in nucleoid organization.

It was suggested that in some bacteria the actin-like protein MreB, apart from affecting cell elongation by governing peptidoglycan assembly, may play a role in ensuring efficient chromosome partitioning (Kruse et al., 2003; Gitai et al., 2005; Kruse and Gerdes, 2005). However, in Streptomyces the main function of MreB appears to be providing stability to the spore wall, which is corroborated by the dense crowding of the inner spore wall by MreB as shown by immuno-electron microscopy (Mazza et al., 2006). Deletion of the paralogous $\mathrm{mb}$ also compromises spore wall integrity (Heichlinger et al., 2011).

\section{Is cell division controlled by the chromosome position?}

With the recruitment of FtsZ by SsgB a basic mechanism of FtsZ localization has been uncovered. What it does not explain is why the distance between the sporulation septa is around $1 \mu \mathrm{m}$. In other words, what controls SsgB localization? SsgB localization is somehow activated by SsgA, but the relationship is yet unclear considering the pleiotropic function of SsgA in cell wall remodelling and the fact that only part of the SsgB foci overlap with those of SsgA (Willemse et al., 2011). Also, SsgA has a much more dynamic localization as SsgB (J. Willemse and G.P. van Wezel, unpublished). It is tempting to suggest that the size of the nucleoid plays a major role in septum spacing. A nucleoid occlusion-like system has not yet been uncovered in Streptomyces, and actinomycetes lack clear homologues of genes encoding proteins inhibiting septa formation over the nucleoid in B. subtilis or E. coli (NOC and SIm; Wu et al., 2009; Wu and Errington, 2011). Electron micrographs of sporulating hyphae showed the constriction of sporulation septa over the not yet fully segregated nucleoids, supporting the view that sporulation-specific cell division is not controlled spatially by the nucleoid position. However, this is apparently challenged by the phenotypes of several mutants. Mutants that lack ssg G frequently 'skip' septa during sporulation but have normal DNA segregation and condensation, thus producing large spores with multiple well-segregated and condensed chromosomes per spore compartment (Noens et al., 2005). This suggests that even in the absence of a physical septum the chromosomes end up at the same location as in wild-type hyphae that undergo normal division. Thus, chromosome condensation may take place independent of the divisome. A similar conclusion was drawn when chromosome segregation was analysed in a non-sporulating strain in which the developmental fts Zp2 promoter had been deleted (Jakimowicz et al., 2006). The presence of a nucleoid occlusion-like system that prevents chromosome damage by the growing septum might explain the observed blockage of septation in hyphae of a topoisomerase depletion strain, where chromosome positioning is highly perturbed (M. Szafran and D. Jakimowicz, unpublished).

Several mutations result in irregularities in both septation and chromosome condensation, including those in the aforementioned dps (Facey et al., 2009) and parA (Jakimowicz et al., 2007). The question that remains unanswered is whether the irregular septation is a direct effect of the disturbed chromosome positioning. Interestingly, in C. crescentus, which like streptomycetes lack a Min system, the ParA homologue MipZ plays a crucial role in septum site localization. MipZ forms a complex with ParB near the origins of replication and the complex then localizes to the cell poles (Thanbichler and Shapiro, 2006). MipZ interferes with FtsZ polymerization, and therefore Z-ring formation is restricted to the mid-cell position, where MipZ concentration is lowest. Thus, MipZ plays a dual role, by positioning the Z-ring and delaying the start of division until chromosome segregation has started (Thanbichler and Shapiro, 2006). The role of the chromosome partitioning proteins in the control of septum site localization in Streptomyces awaits further analysis. 


\section{Concluding remarks and future perspectives}

Further studies should provide more detailed insight into how cell division and chromosome segregation are co-ordinated in streptomycetes. Important mechanisms that are awaiting elucidation include the putative nucleoid occlusion-like mechanism that prevents septum closure over non-segregated chromosomes, the perfect spacing of the septa during sporulation-specific cell division and the role of the seemingly extensive cytoskeleton of streptomycetes in these processes. Such information could emerge from studies of the interaction network between the components of the cell division and chromosome segregation machineries as well as from high-resolution imaging technologies. Correlative microscopy is currently under development that should allow covisualization of fluorescently labelled components (cell wall, proteins, DNA, etc.) at low resolution with high-resolution imaging of cellular ultrastructures, and tomography can be applied to obtain three-dimensional reconstructions of among others septa, the tip growth complex and emerging branches. Such novel structural and molecular insight should aid us in elucidating the many unique aspects of cell division and chromosome segregation in streptomycetes.

\section{Acknowledgements}

We are very grateful to Jolanta Zakrzewska-Czerwińska for comments on the manuscript, to Joe McCormick and Joost Willemse for discussions and sharing unpublished data and to Katherine Celler, Paul Dyson, Roman Koning and Bram Koster for sharing unpublished data. The work is supported by ECHO Grant 700.57.002 from the Netherlands Organization for Scientific Research (NWO) to G. P. v. W.

\section{References}

Adams, D.W., and Errington, J. (2009) Bacterial cell division: assembly, maintenance and disassembly of the $\mathrm{Z}$ ring. Nat Rev Microbiol 7: 642-653.

Alexeeva, S., Gadella, T.W., Jr, Verheul, J., Verhoeven, G.S., and den Blaauwen, T. (2010) Direct interactions of early and late assembling division proteins in Escherichia coli cells resolved by FRET. Mol Microbiol 77: 384-398.

Ausmees, N., Wahlstedt, H., Bagchi, S., Elliot, M.A., Buttner, M.J., and Flardh, K. (2007) SmeA, a small membrane protein with multiple functions in Streptomyces sporulation including targeting of a SpollIE/FtsK-like protein to cell division septa. Mol Microbiol 65: 1458-1473.

Bagchi, S., Tomenius, H., Belova, L.M., and Ausmees, N. (2008) Intermediate filament-like proteins in bacteria and a cytoskeletal function in Streptomyces. Mol Microbiol 70: 1037-1050.

Bennett, J.A., Aimino, R.M., and McCormick, J.R. (2007) Streptomyces coelicolor genes fts $L$ and divIC play a role in cell division but are dispensable for colony formation. J Bacteriol 189: 8982-8992.

Bennett, J.A., Yarnall, J., Cadwallader, A.B., Kuennen, R.,
Bidey, P., Stadelmaier, B., and McCormick, J.R. (2009) Medium-dependent phenotypes of Streptomyces coelicolor with mutations in ftsl or ftsW. J Bacteriol 191: 661664.

Berger, M., Farcas, A., Geertz, M., Zhelyazkova, P., Brix, K., Travers, A., and Muskhelishvili, G. (2010) Coordination of genomic structure and transcription by the main bacterial nucleoid-associated protein HU. EMBO Rep 11: 59-64.

Bernhardt, T.G., and de Boer, P.A. (2005) SImA, a nucleoidassociated, FtsZ binding protein required for blocking septal ring assembly over Chromosomes in E. coli. Mol Cell 18: 555-564.

Bi, E.F., and Lutkenhaus, J. (1991) FtsZ ring structure associated with division in Escherichia coli. Nature 354: 161164.

Bibb, M.J. (2005) Regulation of secondary metabolism in streptomycetes. Curr Opin Microbiol 8: 208-215.

de Boer, P., Crossley, R., and Rothfield, L. (1992) The essential bacterial cell-division protein FtsZ is a GTPase. Nature 359: 254-256.

Burton, B.M., Marquis, K.A., Sullivan, N.L., Rapoport, T.A., and Rudner, D.Z. (2007) The ATPase SpollIE transports DNA across fused septal membranes during sporulation in Bacillus subtilis. Cell 131: 1301-1312.

Chater, K.F. (1972) A morphological and genetic mapping study of white colony mutants of Streptomyces coelicolor. $J$ Gen Microbiol 72: 9-28.

Chater, K.F., and Losick, R. (1997) Mycelial life style of Streptomyces coelicolor A3(2) and its relatives. In Bacteria as Multicellular Organisms. Shapiro, J.A., and Dworkin, M. (eds). New York: Oxford University Press, pp. 149-182.

Chen, Y., Mills, J.D., and Periasamy, A. (2003) Protein localization in living cells and tissues using FRET and FLIM. Differentiation 71: 528-541.

Dalton, K.A., Thibessard, A., Hunter, J.I., and Kelemen, G.H. (2007) A novel compartment, the 'subapical stem' of the aerial hyphae, is the location of a sigN-dependent, developmentally distinct transcription in Streptomyces coelicolor. Mol Microbiol 64: 719-737.

Dedrick, R.M., Wildschutte, H., and McCormick, J.R. (2009) Genetic interactions of $s m c$, ftsK, and parB genes in Streptomyces coelicolor and their developmental genome segregation phenotypes. J Bacteriol 191: 320-332.

Del Sol, R., Mullins, J.G., Grantcharova, N., Flardh, K., and Dyson, P. (2006) Influence of CrgA on assembly of the cell division protein FtsZ during development of Streptomyces coelicolor. J Bacteriol 188: 1540-1550.

Desveaux, D., Allard, J., Brisson, N., and Sygusch, J. (2002) A new family of plant transcription factors displays a novel ssDNA-binding surface. Nat Struct Biol 9: 512-517.

Ditkowski, B., Troc, P., Ginda, K., Donczew, M., Chater, K.F., Zakrzewska-Czerwinska, J., and Jakimowicz, D. (2010) The actinobacterial signature protein ParJ (SCO1662) regulates ParA polymerization and affects chromosome segregation and cell division during Streptomyces sporulation. Mol Microbiol 78: 1403-1415.

Donachie, W.D. (1993) The cell cycle of Escherichia coli. Annu Rev Microbiol 47: 199-230.

Erickson, H.P. (1995) FtsZ, a prokaryotic homolog of tubulin? Cell 80: 367-370.

Erickson, H.P., Anderson, D.E., and Osawa, M. (2010) FtsZ in 
bacterial cytokinesis: cytoskeleton and force generator all in one. Microbiol Mol Biol Rev 74: 504-528.

Errington, J., Daniel, R.A., and Scheffers, D.J. (2003) Cytokinesis in bacteria. Microbiol Mol Biol Rev 67: 52-65.

Facey, P.D., Hitchings, M.D., Saavedra-Garcia, P., Fernandez-Martinez, L., Dyson, P.J., and Del Sol, R. (2009) Streptomyces coelicolor Dps-like proteins: differential dual roles in response to stress during vegetative growth and in nucleoid condensation during reproductive cell division. Mol Microbiol 73: 1186-1202.

Flärdh, K. (2003a) Growth polarity and cell division in Streptomyces. Curr Opin Microbiol 6: 564-571.

Flärdh, K. (2003b) Essential role of DivIVA in polar growth and morphogenesis in Streptomyces coelicolor A3(2). Mol Microbiol 49: 1523-1536.

Flärdh, K., and van Wezel, G.P. (2003) Cell division during growth and development of Streptomyces (Chapter 6). In Recent Developments in Bacteriology. Pandalai, S.G. (ed.). Trivandrum: Transworld Research Network, pp. 71-90.

Flärdh, K., Leibovitz, E., Buttner, M.J., and Chater, K.F. (2000) Generation of a non-sporulating strain of Streptomyces coelicolor A3(2) by the manipulation of a developmentally controlled ftsZ promoter. Mol Microbiol 38: 737749 .

Fleming, T.C., Shin, J.Y., Lee, S.H., Becker, E., Huang, K.C., Bustamante, C., and Pogliano, K. (2010) Dynamic SpollIE assembly mediates septal membrane fission during Bacillus subtilis sporulation. Genes Dev 24: 1160-1172.

Gitai, Z., Dye, N.A., Reisenauer, A., Wachi, M., and Shapiro, L. (2005) MreB actin-mediated segregation of a specific region of a bacterial chromosome. Cell 120: 329-341.

Glazebrook, M.A., Doull, J.L., Stuttard, C., and Vining, L.C. (1990) Sporulation of Streptomyces venezuelae in submerged cultures. J Gen Microbiol 136: 581-588.

Goehring, N.W., and Beckwith, J. (2005) Diverse paths to midcell: assembly of the bacterial cell division machinery. Curr Biol 15: R514-R526.

Goosen, N., and van de Putte, P. (1995) The regulation of transcription initiation by integration host factor. Mol Microbiol 16: 1-7.

Grantcharova, N., Ubhayasekera, W., Mowbray, S.L., McCormick, J.R., and Flardh, K. (2003) A missense mutation in fts $Z$ differentially affects vegetative and developmentally controlled cell division in Streptomyces coelicolor A3(2). Mol Microbiol 47: 645-656.

Grantcharova, N., Lustig, U., and Flärdh, K. (2005) Dynamics of FtsZ assembly during sporulation in Streptomyces coelicolor A3(2). J Bacteriol 187: 3227-3237.

Gueiros-Filho, F.J., and Losick, R. (2002) A widely conserved bacterial cell division protein that promotes assembly of the tubulin-like protein FtsZ. Genes Dev 16: 2544-2556.

Hale, C.A., and de Boer, P.A. (1997) Direct binding of FtsZ to ZipA, an essential component of the septal ring structure that mediates cell division in E. coli. Cell 88: 175-185.

Hamoen, L.W., Meile, J.C., de Jong, W., Noirot, P., and Errington, J. (2006) SepF, a novel FtsZ-interacting protein required for a late step in cell division. Mol Microbiol 59: 989-999.

Hayes, F., and Barilla, D. (2006) The bacterial segrosome: a dynamic nucleoprotein machine for DNA trafficking and segregation. Nat Rev Microbiol 4: 133-143.
Heichlinger, A., Ammelburg, M., Kleinschnitz, E.M., Latus, A., Maldener, I., Flärdh, K., et al. (2011) The MreB-like protein Mbl of Streptomyces coelicolor A3(2) depends on MreB for proper localization and contributes to spore wall synthesis. $J$ Bacteriol 193: 1533-1542.

Hopwood, D.A. (2007) Streptomyces in Nature and Medicine: The Antibiotic Makers. New York: Oxford University Press.

Jakimowicz, D., Mouz, S., Zakrzewska-Czerwinska, J., and Chater, K.F. (2006) Developmental control of a parAB promoter leads to formation of sporulation-associated ParB complexes in Streptomyces coelicolor. J Bacteriol 188: 1710-1720.

Jakimowicz, D., Zydek, P., Kois, A., Zakrzewska-Czerwinska, J., and Chater, K.F. (2007) Alignment of multiple chromosomes along helical ParA scaffolding in sporulating Streptomyces hyphae. Mol Microbiol 65: 625-641.

Jakimowicz, P., Cheesman, M.R., Bishai, W.R., Chater, K.F., Thomson, A.J., and Buttner, M.J. (2005) Evidence that the Streptomyces developmental protein WhiD, a member of the WhiB family, binds a [4Fe-4S] cluster. J Biol Chem 280: 8309-8315.

Jiang, H., and Kendrick, K.E. (2000) Characterization of ssfR and $s s g A$, two genes involved in sporulation of Streptomyces griseus. J Bacteriol 182: 5521-5529.

Judd, E.M., Comolli, L.R., Chen, J.C., Downing, K.H., Moerner, W.E., and McAdams, H.H. (2005) Distinct constrictive processes, separated in time and space, divide Caulobacter inner and outer membranes. J Bacteriol 187: 6874-6882.

Kawamoto, S., and Ensign, J.C. (1995) Isolation of mutants of Streptomyces griseus that sporulate in nutrient rich media: cloning of DNA fragments that suppress the mutations. Actinomycetologica 9: 124-135.

Kawamoto, S., Watanabe, H., Hesketh, A., Ensign, J.C., and Ochi, K. (1997) Expression analysis of the $s s g A$ gene product, associated with sporulation and cell division in Streptomyces griseus. Microbiology 143: 1077-1086.

Keijser, B.J., Noens, E.E., Kraal, B., Koerten, H.K., and van Wezel, G.P. (2003) The Streptomyces coelicolor ssgB gene is required for early stages of sporulation. FEMS Microbiol Lett 225: 59-67.

Kendrick, K.E., and Ensign, J.C. (1983) Sporulation of Streptomyces griseus in submerged culture. J Bacteriol 155: 357-366.

Kim, H.J., Calcutt, M.J., Schmidt, F.J., and Chater, K.F. (2000) Partitioning of the linear chromosome during sporulation of Streptomyces coelicolor A3(2) involves an oriClinked parAB locus. J Bacteriol 182: 1313-1320.

Kois, A., Swiatek, M., Jakimowicz, D., and ZakrzewskaCzerwinska, J. (2009) SMC protein-dependent chromosome condensation during aerial hyphal development in Streptomyces. J Bacteriol 191: 310-319.

Kretschmer, S. (1982) Dependence of the mycelial growth pattern on the individually regulated cell cycle in Streptomyces granaticolor. Z Allg Mikrobiol 22: 335-347.

Kruse, T., and Gerdes, K. (2005) Bacterial DNA segregation by the actin-like MreB protein. Trends Cell Biol 15: 343345.

Kruse, T., Moller-Jensen, J., Lobner-Olesen, A., and Gerdes, K. (2003) Dysfunctional MreB inhibits chromosome segregation in Escherichia coli. EMBO J 22: 5283-5292. 
Kuchibhatla, A., Abdul Rasheed, A.S., Narayanan, J., Bellare, J., and Panda, D. (2009) An analysis of FtsZ assembly using small angle X-ray scattering and electron microscopy. Langmuir 25: 3775-3785.

Kwak, J., Dharmatilake, A.J., Jiang, H., and Kendrick, K.E. (2001) Differential regulation of $\mathrm{fts} Z$ transcription during septation of Streptomyces griseus. J Bacteriol 183: 50925101.

Leonard, T.A., Butler, P.J., and Lowe, J. (2004) Structural analysis of the chromosome segregation protein SpoOJ from Thermus thermophilus. Mol Microbiol 53: 419432.

Leonard, T.A., Butler, P.J., and Lowe, J. (2005a) Bacterial chromosome segregation: structure and DNA binding of the Soj dimer - a conserved biological switch. EMBO J 24: 270-282.

Leonard, T.A., Moller-Jensen, J., and Lowe, J. (2005b) Towards understanding the molecular basis of bacterial DNA segregation. Philos Trans R Soc Lond B Biol Sci 360: 523-535.

Low, H.H., Moncrieffe, M.C., and Lowe, J. (2004) The crystal structure of ZapA and its modulation of FtsZ polymerisation. $J$ Mol Biol 341: 839-852.

McCormick, J.R. (2009) Cell division is dispensable but not irrelevant in Streptomyces. Curr Opin Biotechnol 12: 689698.

McCormick, J.R., and Losick, R. (1996) Cell division gene $f t s Q$ is required for efficient sporulation but not growth and viability in Streptomyces coelicolor A3(2). J Bacteriol 178: 5295-5301.

McCormick, J.R., Su, E.P., Driks, A., and Losick, R. (1994) Growth and viability of Streptomyces coelicolor mutant for the cell division gene ftsZ. Mol Microbiol 14: 243-254.

Marston, A.L., Thomaides, H.B., Edwards, D.H., Sharpe, M.E., and Errington, J. (1998) Polar localization of the MinD protein of Bacillus subtilis and its role in selection of the mid-cell division site. Genes Dev 12: 3419-3430.

Mazza, P., Noens, E.E., Schirner, K., Grantcharova, N., Mommaas, A.M., Koerten, H.K., et al. (2006) MreB of Streptomyces coelicolor is not essential for vegetative growth but is required for the integrity of aerial hyphae and spores. Mol Microbiol 60: 838-852.

Merrick, M.J. (1976) A morphological and genetic mapping study of bald colony mutants of Streptomyces coelicolor. J Gen Microbiol 96: 299-315.

Mistry, B.V., Del Sol, R., Wright, C., Findlay, K., and Dyson, P. (2008) FtsW is a dispensable cell division protein required for Z-ring stabilization during sporulation septation in Streptomyces coelicolor. J Bacteriol 190: 5555-5566.

Miyawaki, A., Llopis, J., Heim, R., McCaffery, J.M., Adams, J.A., Ikura, M., and Tsien, R.Y. (1997) Fluorescent indicators for $\mathrm{Ca}^{2+}$ based on green fluorescent proteins and calmodulin. Nature 388: 882-887.

Muller, W.H., Montijn, R.C., Humbel, B.M., van Aelst, A.C., Boon, E.J., van der Krift, T.P., and Boekhout, T. (1998) Structural differences between two types of basidiomycete septal pore caps. Microbiology 144: 1721-1730.

Muller, W.H., Koster, A.J., Humbel, B.M., Ziese, U., Verkleij, A.J., van Aelst, A.C., et al. (2000) Automated electron tomography of the septal pore cap in Rhizoctonia solani. J Struct Biol 131: 10-18. van Munster, E.B., and Gadella, T.W.J. (2005) Fluorescence Lifetime Imaging Microscopy (FLIM). In Fluorescence Lifetime Imaging Microscopy (FLIM) Microscopy Techniques, Vol. 95. Rietdorf, J. (ed.). Berlin / Heidelberg: Springer, pp. 1301-1303.

Murray, H., and Errington, J. (2008) Dynamic control of the DNA replication initiation protein DnaA by Soj/ParA. Cell 135: 74-84.

Nair, S., and Finkel, S.E. (2004) Dps protects cells against multiple stresses during stationary phase. J Bacteriol 186: 4192-4198.

Navajas, P.L., Rivas, G., Mingorance, J., Mateos-Gil, P., Horger, I., Velasco, E., et al. (2008) In vitro reconstitution of the initial stages of the bacterial cell division machinery. $J$ Biol Phys 34: 237-247.

Noens, E.E. (2007) Control of sporulation-specific cell division in Streptomyces coelicolor. PhD Thesis. Leiden: Leiden University.

Noens, E.E., Mersinias, V., Traag, B.A., Smith, C.P., Koerten, H.K., and van Wezel, G.P. (2005) SsgA-like proteins determine the fate of peptidoglycan during sporulation of Streptomyces coelicolor. Mol Microbiol 58: 929-944.

Noens, E.E., Mersinias, V., Willemse, J., Traag, B.A., Laing, E., Chater, K.F., et al. (2007) Loss of the controlled localization of growth stage-specific cell-wall synthesis pleiotropically affects developmental gene expression in an $s s g A$ mutant of Streptomyces coelicolor. Mol Microbiol 64: 1244-1259.

Pedulla, M.L., and Hatfull, G.F. (1998) Characterization of the mIHF gene of Mycobacterium smegmatis. J Bacteriol 180: 5473-5477.

Pedulla, M.L., Lee, M.H., Lever, D.C., and Hatfull, G.F. (1996) A novel host factor for integration of mycobacteriophage L5. Proc Natl Acad Sci USA 93: 15411-15416.

Pettijohn, D.E. (1988) Histone-like proteins and bacterial chromosome structure. J Biol Chem 263: 12793-12796.

Pichoff, S., and Lutkenhaus, J. (2002) Unique and overlapping roles for ZipA and FtsA in septal ring assembly in Escherichia coli. EMBO J 21: 685-693.

Plocinski, P., Ziolkiewicz, M., Kiran, M., Vadrevu, S.I., Nguyen, H.B., Hugonnet, J., et al. (2011) Characterization of CrgA, a new partner of the Mycobacterium tuberculosis peptidoglycan polymerization complexes. J Bacteriol 193: 3246-3256.

Raskin, D.M., and de Boer, P.A. (1997) The MinE ring: an FtsZ-independent cell structure required for selection of the correct division site in E. coli. Cell 91: 685-694.

RayChaudhuri, D. (1999) ZipA is a MAP-Tau homolog and is essential for structural integrity of the cytokinetic FtsZ ring during bacterial cell division. EMBO J 18: 2372-2383.

Rice, P.A., Yang, S., Mizuuchi, K., and Nash, H.A. (1996) Crystal structure of an IHF-DNA complex: a proteininduced DNA U-turn. Cell 87: 1295-1306.

Ruban-Osmialowska, B., Jakimowicz, D., SmulczykKrawczyszyn, A., Chater, K.F., and ZakrzewskaCzerwinska, J. (2006) Replisome localization in vegetative and aerial hyphae of Streptomyces coelicolor. J Bacteriol 188: 7311-7316.

Salerno, P., Larsson, J., Bucca, G., Laing, E., Smith, C.P., and Flärdh, K. (2009) One of the two genes encoding nucleoid-associated HU proteins in Streptomyces coeli- 
color is developmentally regulated and specifically involved in spore maturation. J Bacteriol 191: 6489-6500.

Scholefield, G., Errington, J., and Murray, H. (2012) Soj/ParA stalls DNA replication by inhibiting helix formation of the initiator protein DnaA. EMBO J 31: 1542-1555.

Schumacher, M.A., Karamooz, E., Zikova, A., Trantirek, L., and Lukes, J. (2006) Crystal structures of T. brucei MRP1/ MRP2 guide-RNA binding complex reveal RNA matchmaking mechanism. Cell 126: 701-711.

Schwedock, J., Mccormick, J.R., Angert, E.R., Nodwell, J.R., and Losick, R. (1997) Assembly of the cell division protein FtsZ into ladder like structures in the aerial hyphae of Streptomyces coelicolor. Mol Microbiol 25: 847-858.

Sevcikova, B., and Kormanec, J. (2003) The ssgB gene, encoding a member of the regulon of stress-response sigma factor sigmaH, is essential for aerial mycelium septation in Streptomyces coelicolor A3(2). Arch Microbiol 180: 380-384.

Thanbichler, M., and Shapiro, L. (2006) MipZ, a spatial regulator coordinating chromosome segregation with cell division in Caulobacter. Cell 126: 147-162.

Toro, E., and Shapiro, L. (2010) Bacterial chromosome organization and segregation. Cold Spring Harb Perspect Biol 2: a000349.

Traag, B.A., and van Wezel, G.P. (2008) The SsgA-like proteins in actinomycetes: small proteins up to a big task. Antonie Van Leeuwenhoek 94: 85-97.

Traag, B.A., Kelemen, G.H., and van Wezel, G.P. (2004) Transcription of the sporulation gene ssgA is activated by the IcIR-type regulator SsgR in a whi-independent manner in Streptomyces coelicolor A3(2). Mol Microbiol 53: 9851000.

Van de Putte, P., Van Dillewijn, J., and Rorsch, A. (1964) The selection of mutants of Escherichia coli with impaired cell division at elevated temperature. Mutat Res 1: 121-128.

Walshaw, J., Gillespie, M.D., and Kelemen, G.H. (2010) A novel coiled-coil repeat variant in a class of bacterial cytoskeletal proteins. J Struct Biol 170: 202-215.

Wang, L., Yu, Y., He, X., Zhou, X., Deng, Z., Chater, K.F., and Tao, M. (2007) Role of an FtsK-like protein in genetic stability in Streptomyces coelicolor A3(2). J Bacteriol 189: 2310-2318.

van Wezel, G.P., and McDowall, K.J. (2011) The regulation of the secondary metabolism of Streptomyces: new links and experimental advances. Nat Prod Rep 28: 1311-1333.

van Wezel, G.P., van der Meulen, J., Kawamoto, S., Luiten, R.G., Koerten, H.K., and Kraal, B. (2000) ssgA is essential for sporulation of Streptomyces coelicolorA3(2) and affects hyphal development by stimulating septum formation. J Bacteriol 182: 5653-5662. van Wezel, G.P., Krabben, P., Traag, B.A., Keijser, B.J., Kerste, R., Vijgenboom, E., et al. (2006) Unlocking Streptomyces spp. for use as sustainable industrial production platforms by morphological engineering. Appl Environ Microbiol 72: 5283-5288.

Wildermuth, H., and Hopwood, D.A. (1970) Septation during sporulation in Streptomyces coelicolor. J Gen Microbio/ 60: 51-59.

Willemse, J., and van Wezel, G.P. (2009) Imaging of Streptomyces coelicolor $\mathrm{A} 3(2)$ with reduced autofluorescence reveals a novel stage of FtsZ localization. PLOS ONE 4: e4242.

Willemse, J., Borst, J.W., de Waal, E., Bisseling, T., and van Wezel, G.P. (2011) Positive control of cell division: FtsZ is recruited by $\mathrm{SsgB}$ during sporulation of Streptomyces. Genes Dev 25: 89-99.

Willemse, J., Mommaas, A.M., and van Wezel, G.P. (2012) Constitutive expression of fts $Z$ overrides the whi developmental genes to initiate sporulation of Streptomyces coelicolor. Antonie Van Leeuwenhoek 101: 619-632.

Wolanski, M., Wali, R., Tilley, E., Jakimowicz, D., Zakrzewska-Czerwinska, J., and Herron, P. (2011) Replisome trafficking in growing vegetative hyphae of Streptomyces coelicolor A3(2). J Bacteriol 193: 1273-1275.

Woldringh, C.L., Mulder, E., Huls, P.G., and Vischer, N. (1991) Toporegulation of bacterial division according to the nucleoid occlusion model. Res Microbiol 142: 309-320.

Wu, L.J., and Errington, J. (2004) Coordination of cell division and chromosome segregation by a nucleoid occlusion protein in Bacillus subtilis. Cell 117: 915-925.

Wu, L.J., and Errington, J. (2011) Nucleoid occlusion and bacterial cell division. Nat Rev Microbiol 10: 8-12.

Wu, L.J., Ishikawa, S., Kawai, Y., Oshima, T., Ogasawara, N., and Errington, J. (2009) Noc protein binds to specific DNA sequences to coordinate cell division with chromosome segregation. EMBO J 28: 1940-1952.

Xu, Q., Traag, B.A., Willemse, J., McMullan, D., Miller, M.D., Elsliger, M.A., et al. (2009) Structural and functional characterizations of SsgB, a conserved activator of developmental cell division in morphologically complex actinomycetes. J Biol Chem 284: 25268-25279.

Yamazaki, H., Ohnishi, Y., and Horinouchi, S. (2003) Transcriptional switch on of $s s g A$ by A-factor, which is essential for spore septum formation in Streptomyces griseus. J Bacteriol 185: 1273-1283.

Yang, Y.H., Song, E., Willemse, J., Park, S.H., Kim, W.S., Kim, E.J., et al. (2012) A novel function of Streptomyces integration host factor (sIHF) in the control of antibiotic production and sporulation in Streptomyces coelicolor. Antonie Van Leeuwenhoek 101: 479-492. 